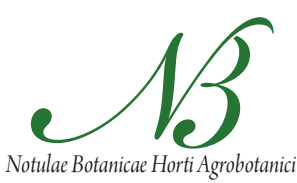

Cluj-Napoca

\title{
QTL Mapping for Days to Flowering under Drought Condition in Rice (Oryza sativa L.) Genome
}

\author{
Supriyo CHAKRABORTY, Zhao-Bang ZENG \\ North Carolina State University, Bioinformatics Research Centre, 1 Lampe Drive, Raleigh, NC 27695, USA; supriyoch_2008@rediffmail.com
}

\begin{abstract}
QTL for days to flowering in rice under drought condition were mapped using a $\mathrm{DH}$ population derived from a cross between a deep-rooted upland adapted japonica genotype 'CT9993-5-10-1-M' and a lowland adapted shallow-rooted moderately drought tolerant indica genotype 'IR62266-42-6-2'. QTL mapping was performed following three different mapping models viz. simple (SIM), composite (CIM) and multiple mapping model (MIM) using WinQTL Cartographer version 2.5.006. SIM located 12 QTL for days to flowering spread over nine chromosomes whereas CIM and MIM each located 5 QTL with a threshold LOD score of 2.5. A comparison of the QTL detected by three different models identified five QTL that were common across at least two models for days to flowering. In MIM analysis, the detected QTL (qHD-1-b) between flanking markers (RG109-ME1014) located on chromosome 1 recorded positive effect (1.4090) but the remaining four QTL had negative effect. The QTL (qHD-3-a) detected between flanking markers (RG104$R G 409$ ) by both MIM and SIM in the present study was also reported earlier as linked with the marker RG104. The five common QTL detected by at least two models could be considered as stable QTL for days to flowering under drought and might be of practical use in marker assisted selection.
\end{abstract}

Keywords: days to flowering, drought, rice, QTL mapping

\section{Introduction}

Rice (Oryza sativa L.) is one of the most important cereal crops in the world and feeds nearly $50 \%$ of the world population. Days to flowering (heading date) in rice is a quantitative trait of agronomic importance. In some agroecosystems rice cultivars with early flowering habit are favored so that they can be fit well in multiple cropping patterns to increase the cropping intensity and crop productivity. The expression of quantitative trait like days to flowering is controlled by several genes with small contributions of each gene to the trait resulting in continuous rather than discrete variation (Liu, 1998). Quantitative traits are highly vulnerable to environmental influences.

In Asia, rice is mostly grown as a rainfed crop due to limited irrigation facilities. The rice crop under rainfed condition often passes through short dry spells resulting in variation of flowering time and low grain yield. The analysis of the quantitative variation of such trait, especially its potential genetic basis, is of prime importance to a plant breeder (Asins, 2002). In fact, plant breeders make use of the genetic variation and the genetic basis of the quantitative trait for formulating an efficient, time-bound breeding program for genetic enhancement of the trait. Because of their features such as large number of genes, small effects and greater influence of environment, the phenotype of the quantitative trait does not provide ample insight into its genotype as against simple monogenic traits (Kearsey, 2002). So molecular marker-mediated genetic analysis is now widely used to dissect such complex quantitative trait into individual Mendelian factors for better understanding of the genetics of the quantitative trait (Pathak and Zhu, 2007).

QTL mapping analysis is one of the molecular marker technologies commonly used for dissecting the complex nature of quantitative traits (Wang et al., 1999). The prime objective of QTL mapping for a quantitative trait is to detect, locate and characterize the QTL (quantitative trait loci) on the chromosome(s). QTLs are the loci/genes controlling quantitative traits. Any chromosomal region associated with a quantitative trait and a marker was defined as QTL (Xu, 2002).

The concept of detecting QTL for quantitative trait loci was first elucidated by Sax (1923) in beans by using pigment markers to analyze genes affecting the seed size in $\mathrm{F}_{2}$ progeny of crosses. Thoday (1960) proposed the idea of using two markers to bracket a region for detecting QTL and was the first known statistical approach. QTL mapping is a combination of the approaches of linkage mapping and statistical genetics to elaborate a quantitative trait at individual gene level in order to draw inferences at population level. QTL mapping analysis is essentially a set of procedures for detecting and locating QTL for a trait. The number of QTL detected depends on the accuracy of the experimental data (both phenotypic and marker data) and the model used for analysis.

Methods commonly used for QTL mapping are simple interval mapping (SIM or IM), composite interval map- 
ping (CIM) and multiple interval mapping (MIM). The principle behind SIM is to test the presence of a QTL at many positions between two marker loci (Lander and Botstein, 1989). CIM performs SIM in the usual way, except that the variance from other QTL is accounted for by including partial regression coefficients from markers in other regions of the genome (Basten et al., 2000). The model of CIM includes one QTL and markers (Kao et al., 1999). It gives more power and precision than SIM because the effects of other QTL are not present as residual variance. CIM can eliminate the bias that would normally be caused by QTL that are linked to the position being tested $(\mathrm{Na}-$ gabhusana et al., 2006).

QTL mapping by MIM model was first proposed by Kao et al. (1999). MIM uses multiple marker intervals simultaneously to construct multiple putative QTL in the model for QTL mapping. Therefore, when compared with SIM and CIM, the MIM tends to be more powerful and precise in detecting QTL. In addition, MIM can readily search for and analyze epistatic QTL and estimate the individual genotypic value and the heritabilities of quantitative traits. On the basis of MIM result, genetic variance components contributed by individual QTL can be estimated and marker assisted selection can be easily performed. Multiple QTL mapping for quantitative trait can be done by using the software R/qtl (Arends et al., 2010).

In rice, QTL for various quantitative traits of agronomic importance have been mapped by different approaches using different populations. A comparison of regression interval mapping (CIM) and MIM for a linked QTL was carried out using simulated dataset of different $\mathrm{F}_{2}$ populations and distinct differences were noticed between the two methods (Mayer, 2005). QTL analysis by both SIM and CIM methods for yield attributing traits in rice was carried out by Cai and Morishima (1998). SIM could detect only three QTL for days to flowering and basic vegetative phase, whereas CIM revealed other significant QTL. The present study was undertaken to detect and locate the significant and common QTL for days to flowering under drought condition in rice using three mapping models namely SIM, CIM and MIM.

\section{Materials and methods}

\section{Plant material}

The plant material used for mapping of the QTL for days to flowering consisted of a population of 154 double haploid $(\mathrm{DH})$ lines derived from a cross between a deeprooted upland adapted japonica rice genotype, 'CT99935-10-1-M' and a lowland indica rice genotype with shallow roots having moderate drought tolerance, 'IR62266-42-62'.

\section{Evaluation for days to flowering}

The lines were grown in $3 \mathrm{mx} 3 \mathrm{~m}$ plots in randomized block design with three replications for two years. Two weeks after transplanting, the fields were drained out and not irrigated again for the rest of the season leading to drought stress. Mean data for days to flowering over two years under moisture stress condition were recorded from 100 lines out of 154 lines grown in Raipur, India and the details of the field experiment are available at http://cropwiki.irri.org/gcp/images/9/9c/IRRI_QTL_Mapping_ workshop.pdf. These data were collected from IRRI, Philippines for the present comparative analysis.

\section{Linkage map}

The genetic linkage map of rice consisting of 280 marker loci including 134 restriction fragment length polymorphisms, 131 amplified fragment length polymorphisms and 15 simple sequence repeats was previously developed from an initial population of $154 \mathrm{DH}$ lines (Zhang et al., 2001). The linkage map covered a map distance of 1602 centimorgans (cM) on the basis of Kosambi function with an average distance of $5.7 \mathrm{cM}$ between adjacent markers. Linkage was declared at LOD score greater than or equal to 2.5 .

\section{QTL analysis}

QTL for days to flowering were detected and mapped on rice chromosomes by performing simple (SIM), composite (CIM) and multiple interval mapping (MIM) analyses using WinQTL Cartographer/version 2.5.006 (Wang et al., 2010) with threshold LOD score of 2.5. The significant threshold LOD score for detection of the QTL was calculated based on 500 permutations at $\mathrm{p} \leq 0.05$ (Churchill and Doerge, 1994) for SIM and CIM. A work speed of $1 \mathrm{cM}$ and a window size of $10 \mathrm{cM}$ were chosen. The number of markers for the background control was set to 5 .

\section{Results and discussion}

Most of the traits of agronomic importance in plants are controlled by many genes having small effect, which are commonly called QTL. QTL are largely influenced by the environment. Mapping of QTL for a trait with a closely linked marker is essential to understand the effect of the QTL on the phenotypic expression of the trait. Considerable progress has been made in the field of QTL mapping in rice (Rahman et al., 2007). To date, a large number of QTL, derived from interspecific crosses and associated with various traits including yield and its components, quality traits, environmental stress tolerance, and disease and insect resistances, have been identified in rice (Aluko et al., 2004; Suh et al., 2005; Thomson et al., 2003).

Rice is mostly grown as a rainfed crop in Asia and the rice crop in the field often passes through intermittent short dry-spells resulting in moisture stress condition, particularly in upland ecosystem. Moisture stress (drought) is a major limiting factor for crop production and it is becoming an increasingly severe problem in many regions of 
60

Tab. 1. QTLs for days to flowering detected by simple, composite and multiple interval mapping models in the DH population of the rice cross 'CT9993-5-10-1M'/'IR62266-42-6-2'

\begin{tabular}{|c|c|c|c|c|c|}
\hline Trait & Model & $\begin{array}{l}\text { No. of } \\
\text { QTLs }\end{array}$ & Chromosome & $\begin{array}{c}\text { Flanking } \\
\text { markers }\end{array}$ & LOD \\
\hline \multirow{22}{*}{$\begin{array}{l}\text { Days to } \\
\text { flowering }\end{array}$} & \multirow{12}{*}{ SIM } & 1 & 1 & RM212-ME418 & 5.7 \\
\hline & & \multirow[t]{2}{*}{2} & \multirow[t]{2}{*}{2} & C1408-EM1110 & 4.7 \\
\hline & & & & TGMSP2-K706 & 3.6 \\
\hline & & 1 & 3 & RG104-RG409 & 3.9 \\
\hline & & 1 & 5 & C119-EMP210 & 3.5 \\
\hline & & 1 & 7 & ME215-EM1511 & 5.4 \\
\hline & & \multirow[t]{3}{*}{3} & \multirow[t]{3}{*}{8} & ME28-EM166 & 3.1 \\
\hline & & & & ME84-R1394A & 4.6 \\
\hline & & & & RZ997-EM185 & 6.3 \\
\hline & & 1 & 9 & K985-RM215 & 3.5 \\
\hline & & 1 & 11 & EM188-G257 & 3.3 \\
\hline & & 1 & 12 & ME29-ME415 & 5.0 \\
\hline & \multirow{5}{*}{ CIM } & \multirow{5}{*}{$\begin{array}{l}1 \\
1 \\
1 \\
2\end{array}$} & \multirow{5}{*}{$\begin{array}{l}1 \\
2 \\
7 \\
8\end{array}$} & RM212-ME418 & 3.5 \\
\hline & & & & C1408-EM1110 & 3.9 \\
\hline & & & & ME71-EM165 & 3.5 \\
\hline & & & & EM141-RZ997 & 3.8 \\
\hline & & & & RZ997-EM185 & 4.6 \\
\hline & \multirow{5}{*}{ MIM } & \multirow{2}{*}{2} & \multirow[t]{2}{*}{1} & RM212-ME418 & 4.6 \\
\hline & & & & RG109-ME1014 & 5.2 \\
\hline & & \multirow{3}{*}{$\begin{array}{l}1 \\
2\end{array}$} & \multirow{3}{*}{$\begin{array}{l}3 \\
5\end{array}$} & RG104-RG409 & 2.8 \\
\hline & & & & C119-EMP210 & 2.8 \\
\hline & & & & RG164-RG573 & 3.0 \\
\hline
\end{tabular}

Tab. 2. Common QTLs detected for days to flowering by two or more QTL mapping models in the $\mathrm{DH}$ population of the rice cross 'CT9993-5-10-1-M'/'IR62266-42-6-2'

\begin{tabular}{ccccc}
\hline Trait & QTL Models & Chromosome & $\begin{array}{c}\text { No. of } \\
\text { QTLs }\end{array}$ & $\begin{array}{c}\text { Flanking } \\
\text { markers }\end{array}$ \\
\hline \multirow{5}{*}{ Days to } & SIM, CIM, MIM & 1 & 1 & RM212-ME418 \\
flowering & SIM, CIM & 2 & 1 & C1408-EM1110 \\
& SIM, MIM & 3 & 1 & RG104-RG409 \\
& SIM, MIM & 5 & 1 & C119-EMP210 \\
& SIM, CIM & 8 & 1 & RZ997-EM185 \\
& & & & \\
\hline
\end{tabular}

the world (Passioura, 2007). Drought stress induces several physiological, biochemical and molecular responses in crop plants, which would help them to adapt to such limiting environmental conditions (Arora et al., 2002). It inhibits the photosynthesis of plants, causes changes in chlorophyll contents and components, and also causes damage to the photosynthetic apparatus (Escuredo et al., 1998). Drought tolerant crop plants usually possess one or more of the drought tolerant mechanisms, such as escape, avoidance, tolerance and recovery (Asch et al., 2005). It has been estimated that nearly $50 \%$ of the world rice production is more or less affected by drought (Bouman et al., 2005). Moisture stress brings about several developmental changes in the rice plant, often leading to early flowering, shortening of life cycle and invariably low grain yield. Shortening of life cycle (generally defined by short growth duration or early flowering) constitutes an attribute of "drought escape", a mechanism to get rid of drought stress, by the plants (Allah, 2010). Rice is very sensitive to moisture stress during flowering resulting in high floret sterility. For rice genetic improvement, it is thus essential to understand the genetic control of flowering under moisture stress condition in order to develop rice cultivars suitable for such condition.

In the present study, days to flowering ranged from 88.5 to 119.5 days with a mean value of 99.6 days and a coefficient of variation of $6.06 \%$. The number of QTL for days to flowering detected by SIM, CIM and MIM models are presented along with the chromosome number, flanking markers and peak LOD values (Tab. 1).

Simple interval mapping detected a total of 12 QTL for days to flowering under drought condition spread over nine chromosomes (Tab. 1). All the detected QTL had a LOD score equal to or more than 2.5 (Fig. 1). Li et al. (2003) detected QTL for days to flowering on rice chromosome 1, 2, 3, 5, 8, 9, 11 and 12 from the rice $\mathrm{DH}$ population of IR-64/Azucena. Yamamoto et al. (2000) located QTL for days to flowering on chromosome 7 in the $\mathrm{BC}_{4} \mathrm{~F}_{2}$ population of Nipponbare / Kasalath.

Composite interval mapping in the present study detected five QTL for days to flowering i.e. one QTL each on chromosome 1, 2 and 7 and two on chromosome 8 (Tab. 1). The locations of the QTL are presented in Fig. 2. The QTL on chromosome 1 between flanking markers RM 212- ME 418 was commonly detected by SIM, CIM and MIM models.

Like CIM, multiple interval mapping also detected five QTL for days to flowering i.e. 2 QTL each on chromosome 1 and 5 but 1 QTL only on chromosome 3 (Tab. 1). The locations of the detected QTL are presented in Fig. 3. The QTL (qHD-3-a) detected by both MIM and SIM

Tab. 3. Estimates of QTL effects for days to flowering and percent variance explained by each QTL in MIM model in the DH population of the rice cross 'CT9993-5-10-1-M'/'IR62266-42-6-2'

\begin{tabular}{ccccccc}
\hline $\begin{array}{c}\text { QTL name } \\
\text { (given) }\end{array}$ & Chromosome & Flanking markers & QTL effect & $\begin{array}{c}\text { \% Genetic } \\
\text { variance }\end{array}$ & $\begin{array}{c}\text { \% Phenotypic } \\
\text { variance }\end{array}$ & Gene action \\
\hline qHD-1-a & 1 & RM212-ME418 & -2.56 & 42.63 & 17.2 & Additive \\
qHD-1-b & 1 & RG109- ME1014 & 1.41 & 1.82 & 0.7 & Additive \\
qHD-3-a & 3 & RG104- RG409 & -2.50 & 30.18 & 12.2 & Additive \\
qHD-5-a & 5 & C119- EMP210 & -1.29 & 18.24 & 7.4 & Additive \\
qHD-5-b & 5 & RG164- RG573 & -0.83 & 7.13 & 2.9 & Additive \\
\hline
\end{tabular}




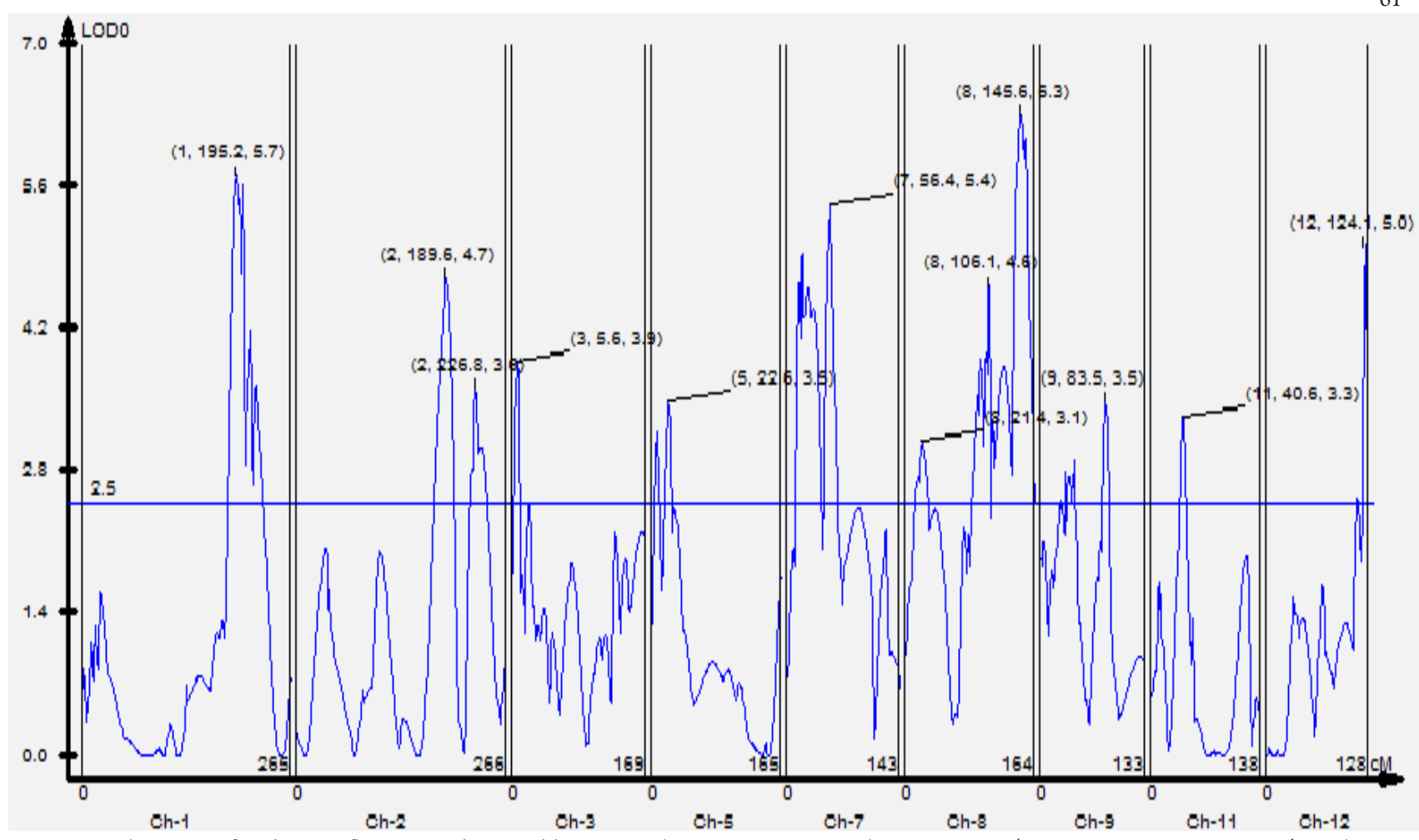

Fig. 1. Twelve QTL for days to flowering detected by interval mapping on rice chromosomes $(1,2,3,5,7,8,9,11 \& 12)$ in the $\mathrm{DH}$ population of the rice cross 'CT9993-5-10-1-M'/'IR62266-42-6-2'

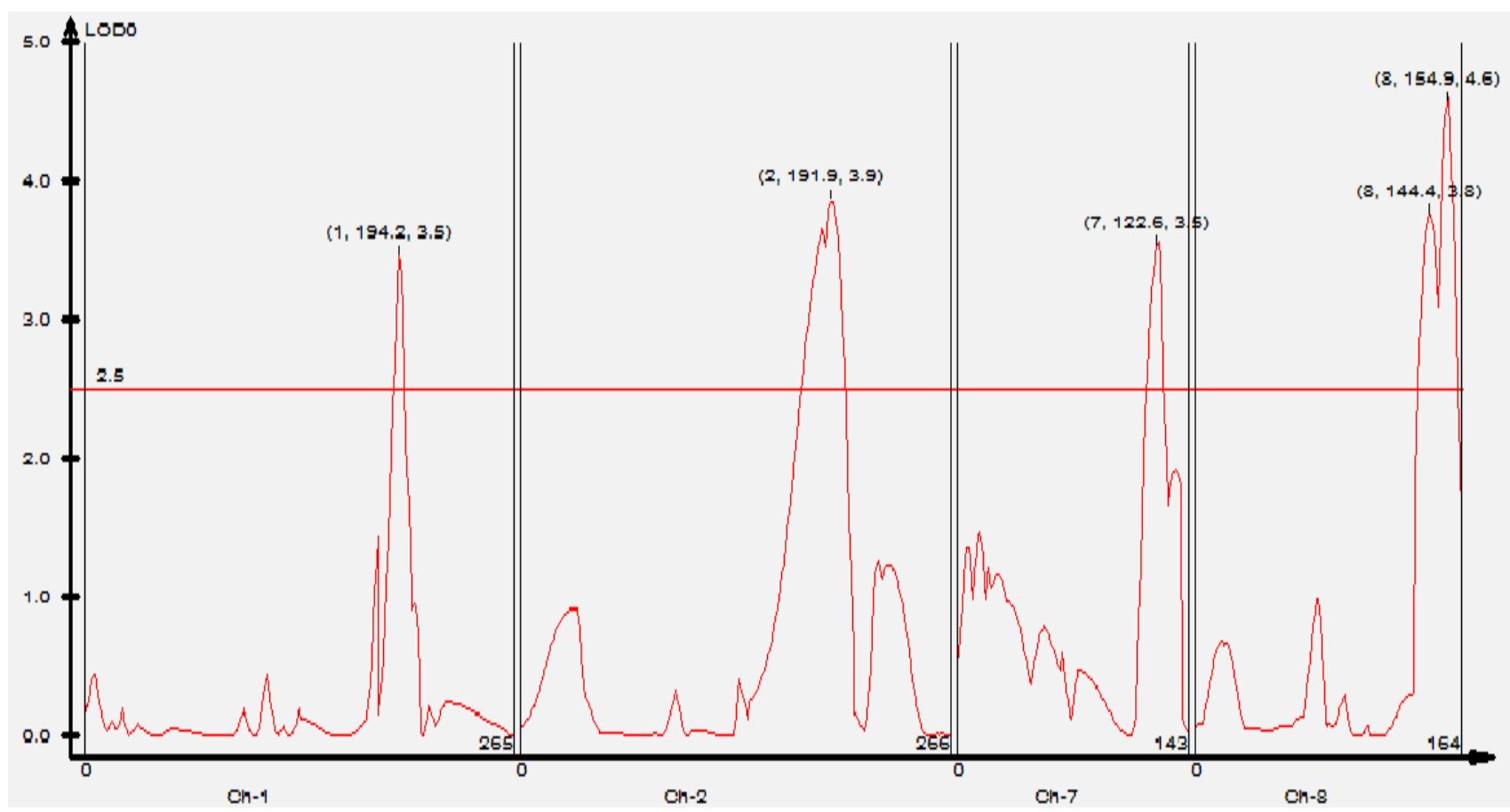

Fig. 2. Five QTL for days to flowering detected by composite interval mapping on rice chromosomes $(1,2,7 \& 8)$ in the DH population of the rice cross 'CT9993-5-10-1-M'/'IR62266-42-6-2'

models in the present study between the flanking markers (RG104 - RG409) on chromosome 3 was also reported earlier as linked with the marker RG104 by Li et al. (2003) in the IR64 / Azucena DH population.
The QTL which were commonly detected by two or more models are presented in Tab. 2. In MIM analysis, the genetic and the phenotypic variance for days to flower- 


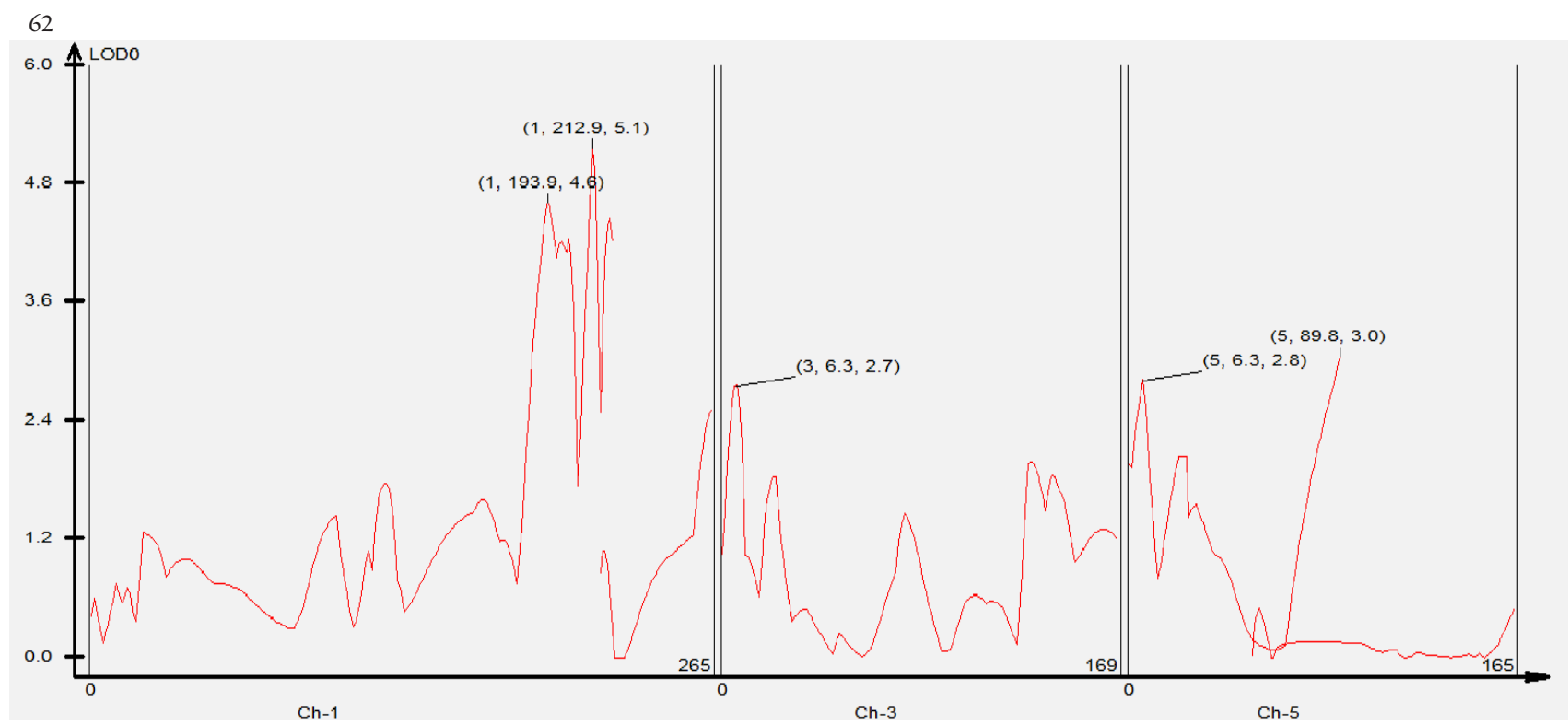

Fig. 3. Five QTL for days to flowering detected by multiple interval mapping model on rice chromosomes $(1,3 \& 5)$ in the DH population of the rice cross 'CT9993-5-10-1-M'/'IR62266-42-6-2'

ing were estimated as 14.59 and 36.12 , respectively with a heritability estimate of $40.39 \%$.

The estimates of QTL effects and the amount of genetic and phenotypic variance explained by each QTL in MIM model for days to flowering are presented in Tab. 3. The QTL (qHD-1-b) located on chromosome 2 recorded positive effect whereas the remaining QTL recorded negative effect (i.e. desirable for developing early flowering variety with short duration to fit into multiple cropping patterns). The sum of the phenotypic variance explained by all the QTL was $40.4 \%$ i.e. the heritability estimate of the trait. In other words, the detected QTLs for days to flowering could explain only $40.4 \%$ of the variation in the trait under drought condition.

The identification of almost entirely different sets of QTL for days to flowering in different environmental conditions is not at all implausible according to the concepts of developmental genetics (Pathak and Jhu, 2007). Development is sequential and hierarchical in nature that generates integrative networks of inter relationships within and between levels of organization. Developmental genetic theories suggest that, because of its sequential and hierarchical nature, development is epigenetic and involves cascades of interactions among directly and indirectly acting controlling factors and these interactions may vary during ontogeny (Atchley, 1990). Thus it was highly expected that several QTL, though not necessarily entirely different set of QTL, would be detected for days to flowering in the moisture stress environment of the present study.

\section{Conclusions}

The present study provides insight into the genetic control of days to flowering in rice under moisture stress condition. With the same number and set of markers and genotypes used, SIM located 12 QTL for days to flowering spread over nine chromosomes whereas CIM and MIM each located 5 QTL with a threshold LOD score of 2.5. However, considerable resemblance among SIM, CIM and MIM was observed for the common QTL detected by at least two models with respect to the position of QTL on chromosomes. In MIM analysis, the detected QTL (qHD-1-b) between flanking markers ( $R G 109$ ME1014) located on chromosome 1 recorded positive effect (1.4090) but the remaining four QTL had negative effect. The QTL (qHD-3-a) detected between flanking markers (RG104 - RG409) by both MIM and SIM in the present study was also reported earlier as linked with the marker RG104. A set of five QTL that were common across at least two models were detected and located in the present study. The detection of such common QTL for a quantitative trait of agronomic importance by different mapping models helps in designing strategies to improve the trait by targeting such QTL through marker-assisted selection.

\section{Acknowledgements}

The first author is thankful to the Department of Biotechnology, Ministry of Science \& Technology, Government of India, New Delhi for providing him the DBT Overseas Associateship for North Eastern Region (No. $\mathrm{BT} / 05 / \mathrm{NE} / 2009)$ to undertake the present research work.

\section{References}

Allah AA (2010). Development of some high yielding rice lines tolerant to drought stress conditions. J Med Pl Res 4(7):528535.

Aluko GC, Martinez J, Tohme C, Castano C, Bergman C, Oard 
JH (2004). QTL mapping of grain quality traits from the interspecific cross Oryza sativa x O. glaberrima. Theor Appl Genet 109:630-639.

Arends D, Prins P, Jensen RC, Broman KW (2010). R/qtl: high-throughput multiple QTL mapping. Bioinformatics 26(23):2990-2992.

Arora A, Sairam RK, Srivastava GC (2002). Oxidative stress and antioxidative systems in plants. Curr Sci 82:1227-1238.

Asch F, Dingkuhn M, Sow A, Audebert A (2005). Droughtinduced changes in rooting patterns and assimilate partitioning between root and shoot in upland rice. Field Crops Res 93(2/3):223-236.

Asins MJ (2002). Present and future of QTL analysis in plant breeding. Plant Breed 121:281-286.

Atchley WR(1990). Heterochrony and morphological change: a quantitative genetic perspective. Seminars in Developmental Biology 1:289-297.

Basten CJ, Weir BS, Zeng ZB (2000). QTL Cartographer. North Carolina State University, USA.

Bouman BAM, Peng S, Castaoeda AR, Visperas RM (2005). Yield and water use of irrigated tropical aerobic rice systems. Agricultural Water Management 74(2):87-105.

Cai HW, Morishima H (1998). Mapping QTLs for heading behavior using RI population derived from a cross between wild and cultivated rice strains. Rice Genet Newsl 15:144145.

Churchill GA, Doerge RW (1994). Empirical threshold values for quantitative trait mapping. Genetics 138:963-971.

Escuredo IP, Arrese-Igor C, Becana M (1998). Oxidative damage in pea plants exposed to water deficit or paraquat. Plant Physiol 116:173-181.

Kao CH, Zeng Z, Teasdale K (1999). Multiple interval mapping for quantitative trait loci. Genetics 152:1203-1216.

Kearsey MJ (2002). QTL Analysis: Problems and Possible Solutions. Quantitative Genetics, Genomics and Plant Breeding. Kang MS (Ed.). CABI Publishing, p. 45-57.

Lander E, Botstein D (1989). Mapping Mendelian factors underlying quantitative traits using RFLP linkage maps. Genetics 121:185-199.

Li Z, Yu SB, Laffittle H, Huang N, Courtois B, Hittalmani H, Lin GF, Wang G, Sashidar H, Zhuang J, Zhang K, Singh VP, Sidhu JS, Khush GS (2003). QTL x environmental interaction in rice. I. Heading date and plant height. Theor Appl Genet 108:141-153.

Liu BH (1998). Statistical Genomics: Linkage Mapping and QTL Analysis, CRC Press, USA, p:611.

Mayer M (2005). A comparison of regression interval mapping and multiple interval mapping for linked QTL. Heredity 94:599-605.
Nagabhushana K, Mane SP, Hillalmani S (2006). Comparative studies on QTL mapping by simple interval mapping and composite interval mapping models for selected growth and yield traits in rice (Oryza sativa L). Indian J Crop Sci 1(12):97-101.

Passioura JB (2007). The drought environment: physical, biological and agricultural perspectives. J Exp Bot 58(2):113117.

Pathak PK, Zhu J (2007). QTLs for response to low temperature stress during seedling growth in rice. Indian $\mathrm{J}$ Genet 67(4):337-346.

Rahman ML, Chu SH, Choi M, Qiao YL, Jiang W, Piao R, Khanam S, Chou Y, Jeung J, Jena KK, Koh H (2007). Identification of QTLs for some agronomic traits in rice using an introgression line from Oryza minuta. Mol Cells $34(1): 16-26$.

Sax K (1923). The association of size differences with seed coat pattern and pigmentation in Phaseolus vulgaris. Genetics 8:552-560.

Suh J, Ahn SN, Cho YC, Kang KH, Choi IM (2005). Mapping for QTLs for yield traits using an advanced backcross population from a cross between Oryza sativa and $O$. glaberrima. Korean J Breed 37:214-220.

Thoday JM (1960). Location of polygenes. Nature 191:368370.

Thomson MJ, Tai T, McClung AM, Lai H, Hinga ME, Lobos KB, Xu Y, Martinez CP, McCouch SR (2003). Mapping quantitative trait loci for yield components and morphological traits in an advanced backcross population between Oryza rufipogon and the Oryza sativa cultivar Jefferson. Theor Appl Genet 107:479-493.

Wang D, Zhu J, Li Z, Paterson AH (1999). User manual for QTL Mapper v1.1. A computer software for mapping quantitative trait loci with main effects, epistatic effects and QTL $x$ Environment interactions. Zhejiang University, China.

Wang S, Basten CJ, Zeng ZB (2010). Windows QTL Cartographer 2.5, Department of Statistics, North Carolina State University, Raleigh, NC, USA (Available from http:// statgen.ncsu.edu/qticart/WQTLCart.htm).

Xu J (2002). Global view of QTL. Quantitative Genetics, Genomics and Plant Breeding, Kang MS (Eds.). CABI Publishing.

Yamamoto T, Lin H, Sasaki T, Yano M (2000). Identification of heading date QTL Hd6 and characterization of its epistatic interaction with $\mathrm{Hd} 2$ in rice using advanced backcross progeny. Genetics 154:885-891.

Zhang J, Zheng HG, Aarti A, Pantuwan G, Nguyen TT, Tripathy JN, Sarial AK, Robin S, Babu RC, Nguyen BD (2001). Locating genomic regions associated with components of drought resistance in rice: comparative mapping within and across species. Theor Appl Genet 103:19-29. 